\title{
Dependência Térmica em Dispositivos Ópticos Baseados em Fibras Ópticas com Dispersão Ultra-Plana
}

\author{
J. Patrocínio da Silva, V. F. Rodríguez-Esquerre, D. S. Bezerra e H. E. Hernández-Figueroa
}

\begin{abstract}
Resumo-Sabe-se que o indice de refração dos materiais usados na fabricação de fibras ópticas podem sofrer variações com a temperatura. Neste caso, pode-se esperar uma variação dos parâmetros relativos às fibras ópticas bem como nos dispositivos baseados nestas. Essa característica poderá conduzir a projetos de sensores de temperatura ou dispositivos térmicos através da maximização ou compensação desses efeitos. Neste trabalho, analisam-se as características de acoplamento para um acoplador direcional baseado em duas fibras com dispersão ultra-plana. As fibras estão separadas por uma pequena distância, $d$, com propósito de monitorar a transferência de energia entre os seus núcleos. Para esta análise, uma eficiente formulação vetorial baseada no método dos elementos finitos foi usada.
\end{abstract}

Palavras-Chave-Dispersão cromática, acoplador óptico, temperatura, dispositivos fotônicos.

Abstract-It is well known that refractive indexes of materials used for fabricating optical fibers are affected by variations of temperature. In this way, it can be expected a dependence on the temperature for several parameters related with optical fibers and devices based on them. The understanding of this dependence can lead to the design of temperature sensors or thermal devices through the maximization or the compensation of these effects, respectively. In this work we proposed and analyzed the coupling characteristics for a directional coupler based on two ultralow dispersion fibers separated by a small distance for power monitoring purposes. An efficient vectorial finite element method has been used for this purpose.

Keywords-Chromatic dispersion, optical coupler, temperature, photonic devices.

\section{INTRODUÇÃO}

As fibras ópticas apresentam importantes características, que as transformam em um excelente meio de transmissão para pequenas e longas distâncias. Dentre estas, podemos destacar a dispersão cromática que é um parâmetro importante e deve ser cuidadosamente analisado durante o desenvolvimento da estrutura. Neste aspecto, vários perfis de índices de refração têm sido analisados, inclusive considerando alguns tipos de cristais fotônicos, os quais apresentam baixa dispersão cromática com grandes larguras de banda [1]-[3].

Neste trabalho, o método dos elementos finitos em conjunto com uma análise modal é usado para analizar, em diferentes

J. Patrocínio da Silva e D. S. Bezerra, Departamento de Ciências Ambientais e Tecnológicas - DCAT, Universidade Federal Rural do SemiÁrido, Mossoró-RN, Brasil. V. F. Rodríguez-Esquerre, Departamento de Tecnologia Eletro-Eletrônica, DTEE, Instituto Federal da Bahia, IFET-BA, Brasil. H. E. Hernández-Figueroa, Departamento de Microondas e Óptica, Univesidade Estadual de Campinas, DMO-FEEC, UNICAMP, Campinas, Brasil. E-mails: patroc@ufersa.edu.br, diego.bezerra@mcc.ufersa.edu.br, vitaly@cefetba.br, hugo@dmo.fee.unicamp.br. temperaturas, a dispersão cromática em fibras com dispersão cromática ultra-plana. Através do método da propagação vetorial de feixes foram analisado os efeitos térmicos no comprimento de acoplamento num acoplador baseado nas fibras previamente descritas. Os coeficientes de Sellmeier, com dependência da temperatura [4],[5], foram usados para modelar, com precisão, os índices de refração da sílica. Sabese que a sílica representa uma classe de material bastante usado na confecção de dispositivos ópticos, por ser um material comumente usado em vários comprimentos de onda e em várias temperaturas. A estrutura proposta é formada pela inclusão de um buraco circular de raio muito pequeno, introduzido no centro do núcleo de uma fibra óptica convencional, como mostra a Fig. 1a.

Aqui, esse novo e simples tipo de fibra, também é aplicado para constituir um acoplador composto por duas destas fibras separadas por uma distância $d$, constante ao longo da direção de propagação. A estrura proposta para o acoplador é mostrada na Fig. 1b e mais uma vez, o método dos elementos finitos em conjunto com o método da propagação vetorial de feixes, mostrou-se extremamente eficiente na análise destes tipos de estruturas fotônicas.

Na próxima seção, será feita uma rápida explanação sobre a formulação com o método dos elementos finitos e a aplicação dos coeficientes de Sellmeier dependentes da temperatura. Na seção III são apresentados resultados numéricos para uma fibra com dispersão cromática ultraplana mostrando sua sensibilidade a variações de temperaturas. Nesta seção, também serão mostradas as características de acoplamento para a estrutura mostrada na Fig 1b e finalmente, na seção IV, serão apresentadas as principais conclusões obtidas neste trabalho.

\section{Formulação POR Elementos Finitos E COEFICIENTES DE SELLMEIER}

O método dos elementos finitos é amplamente reconhecido como uma poderosa ferramenta numérica para análise de dispositivos ópticos [5]-[10]. A aproximação por elementos finitos vetoriais para análise das características de propagação em fibras ópticas com alta precisão é mostrado em [3]. Em sistemas de comunicações ópticas, o conhecimento da dispersão cromática $D$ torna-se extremamente importante para determinar a qualidade da transmissão do pulso, devido este fator ser diretamente responsável pela largura do pulso. Por outro lado, $D$ é proporcional a derivada segunda da constante de propagação ou índice de refração efetivo $n_{\text {eff }}$, desta forma, 
uma ferramenta numérica altamente precisa, como o método dos elementos finitos [8], torna-se necessário para obter o $n_{\text {eff }}$. Aqui, além da aplicação do método numérico, os coeficientes de Sellmeier com dependência da temperatura, foram usados na formulação para analisar a dispersão em diferentes temperaturas.

Em síntese, a formulação é obtida, partindo-se da equação vetorial de Helmholtz em duas dimensões e considerando camadas perfeitamentes casadas (PMLs) para evitar reflexões indejadas. Desta forma, pode-se escrever:

$$
\nabla \times(\overline{\bar{k}} \nabla \times \vec{H})-k_{0}^{2} \vec{H}=0
$$

onde $\overline{\bar{k}}=1 / \overline{\bar{\varepsilon}}$ e $\overline{\bar{\varepsilon}}$ representa o tensor permissividade relativa. Considerando o meio dielétrico com anisotropia transversal e definindo $\hat{u}_{x}, \hat{u}_{y}$ e $\hat{u}_{z}$ associados com as direções $x, y$ e $z$ respectivamente, $\overline{\bar{\varepsilon}}$ pode ser escrito como $\overline{\bar{\varepsilon}}=\varepsilon_{x x} \hat{u}_{x} \hat{u}_{x}+$ $\varepsilon_{x y} \hat{u}_{x} \hat{u}_{y}+\varepsilon_{y x} \hat{u}_{y} \hat{u}_{x}+\varepsilon_{z z} \hat{u}_{z} \hat{u}_{z}$. Após algumas manipulações algébricas e assumindo que tanto o meio como os campos variam muito lentamente ao longo da direção de propagação, $z$ [10], a equação (1) poderá ser reescrita como:

$$
\begin{gathered}
\overline{\bar{k}}_{a} \frac{\partial^{2} \vec{h}_{T}}{\partial z^{2}}-2 \gamma \overline{\bar{k}}_{a}-\overline{\bar{k}}_{b} \nabla_{T}\left(\nabla_{T} \cdot \vec{h}_{T}\right)-\nabla_{T} \times k_{z z} \nabla_{T} \\
\times \vec{h}_{T}+\left(\overline{\bar{k}}_{c}+\gamma^{2} \overline{\bar{k}}_{a}\right) \vec{h}_{T}=0
\end{gathered}
$$

Consequentemente, o tensor tranversal da equação (1) pode ser definido como:

$$
\begin{gathered}
\overline{\bar{k}}_{a}=\left[\begin{array}{cc}
k_{y y} & -k_{y x} \\
-k_{x y} & k_{x x}
\end{array}\right] \\
\overline{\bar{k}}_{b}=\gamma^{-1} \frac{\partial \overline{\bar{k}}_{a}}{\partial z}-\overline{\bar{k}}_{a} \\
\overline{\bar{k}}_{c}=\overline{\bar{k}}_{0}^{2}-\gamma^{-1} \frac{\partial \overline{\bar{k}}_{a}}{\partial z} \\
\nabla_{T}=\hat{u}_{x} \alpha_{x} \frac{\partial}{\partial x}+\hat{u}_{y} \alpha_{y} \frac{\partial}{\partial y}
\end{gathered}
$$

onde $\alpha_{x}$ e $\alpha_{y}$ são parâmetros relatimos as PMLs ou ao meio com perdas virtuais. Desde que a propagação ocorra ao longo da direção $z$, o parâmetro $\alpha_{z}$ é igual a unidade e demais parâmetros das PMLs têm que ser determinados de tal forma, que a impedância da onda possa ser contínua através das interfaces formadas entre o meio interno do domínio computacional a as PMLs. Isto leva a um perfeito casamento sobre as interfaces e as radiações indesejáveis são evitadas. Ou seja, simula-se uma propagação no espaço livre em um domínio computacional reduzido, sem occorrer reflexões nas paredes da janela computacional considerada. Deste modo, os demais parâmetros das PMls são dados em função do parâmetro $S=1-j\left(3 c / 2 \omega_{0} n d\right)(\rho / d)^{2} \ln (1 / R)$ [10], onde $\omega_{0}$ representa a frequiência angular, $d$ é espessura das PMLs, $n$ é o índice de refração do meio adjacente, $\rho$ é a distância da parede interna da PML $R$ é o coeficente de reflexão e $c$ é a velocidade da luz no espaço livre.
Aplicando o metodo dos elementos finitos para a variação transversal em (2), obtém-se a seguinte equação diferencial.

$$
[M] \frac{\partial^{2} \vec{h}_{T}}{\partial z^{2}}-2 \gamma[M] \frac{\partial \vec{h}_{T}}{\partial z}+\left([K]+\gamma^{2}[M]\right)\left\{\vec{h}_{T}\right\}=\{0\}
$$

Onde $\left\{\vec{h}_{T}\right\}$ representa um vetor coluna que contém as componentes desconhecidas $h x j$ e $h y j,\{0\}$ é o vetor coluna nulo e $[M]$ e $[K]$ são denominadas matrizes globais definidas em [10]. Aplicando a aproximação de Padé $(1,1)$ [10], na equação (7), a mesma poderá ser reescrita da seguinte forma:

$$
[\widetilde{M}] \frac{d\left\{\vec{h}_{T}\right\}}{d z}+[K]\left\{\vec{h}_{T}\right\}=\{0\}
$$

onde $[\widetilde{M}]=[M]-\frac{1}{4 \gamma^{2}}\left([K]+\gamma^{2}[M]\right)$. Finalmente, o método das disferenças finitas é aplicado, transformando o problema em um sistema de equações algébricas do tipo:

$$
\begin{gathered}
([\widetilde{M}(z)]+\theta \Delta z[K(z)])\left\{\vec{h}_{T}(z+\Delta z)\right\}= \\
\left([\widetilde{M}(z)]-(1-\theta) \Delta z[K(z)]\left\{\vec{h}_{T}(z)\right\}\right)
\end{gathered}
$$

onde $\Delta z$ é o passo de propagação ao longo da direção $z$ e $(0 \leq \theta \leq 1)$ é introduzido para controle de estabilidade do método. Estudos realizados sobre estabilidade confirmam que este método é incondicionalmente estável para $0,5 \leq \theta \leq 1$. Para $\theta=0,5$ em (9) tem-se o já conhecido algoritmo de Crank Nicholson [11].

Para melhorar a precisão do método usado, o índice de refração de referência é recalculado a cada passo de propagação, seguindo a prescrição dada em [10], como a seguir:

$$
n_{\text {eff }}^{2}=\operatorname{Re}\left[\frac{\left\{\vec{h}_{T}(z)\right\}^{\dagger}[K(z)]\left\{\vec{h}_{T}(z)\right\}}{k_{0}^{2}\left\{\vec{h}_{T}(z)\right\}^{\dagger}[M(z)]\left\{\vec{h}_{T}(z)\right\}}\right]
$$

onde $\dagger$ representa o complexo conjugado e transposto.

Em adição, é importante ressaltar que o perfil de índice de refração $n(r, \lambda)$ de uma fibra óptica pode ser escrito como $n(r, \lambda)=\eta(r) n_{s}(\lambda)$, onde $n_{s}(\lambda)$ é o índice de refração da sílica pura e $\eta(r)$ é o índice de refração normalizado, o qual é função apenas da coordenada radial. Aqui, as constantes de Sellmeier dependentes da temperatura [1]-[3], para sílica, são consideradas. Desta forma, a dispersão do material é incluida diretamente nos nossos cálculos. Os coeficientes de Sellmeier foram usados porque eles podem ajustar-se as medidas experimentais dos índices de refração da sílica. Para sílica fundida $\left(\mathrm{SiO}_{2}\right)$, a equação de Sellmeier é da forma:

$$
n^{2}=A+B /\left(1-C / \lambda^{2}\right)+D /\left(1-E / \lambda^{2}\right)
$$

onde $A, B, C$ e $D$ são os coeficientes de temperatura de primeira ordem e $E$ é uma constante e seus valores são dados por [2], [12]-[13]: $A=1,31552+6,90754 \times 10^{-6} \mathrm{~T} ; B$ $=78,8404+2,35835 \times 10^{-5} \mathrm{~T} ; C=110,199+5,84758$ x $10^{-7} \mathrm{~T} ; D=0,91316+5,48368 \times 10^{-7} \mathrm{~T}$ e $E=100$. Aqui, $\mathrm{T}$ representa a temperatura em graus celsius. Essas constantes podem ser usadas no intervalo $\left(26^{\circ} \mathrm{C}<\mathrm{T}<471^{\circ} \mathrm{C}\right.$ e $\lambda>1,1 \mu \mathrm{m})$. 


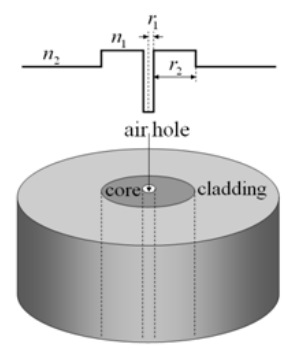

(a)

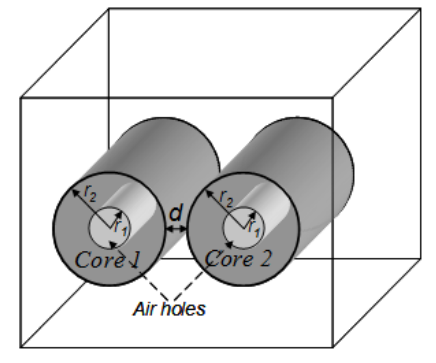

(b)
Fig. 1. (a) Perfil de índice de refração da fibra óptica com buraco de ar no núcleo e (b) Acoplador constituido a partir de duas fibras deste tipo.

\section{RESUlTADOS NUMÉRICOS}

Para verificar o efeito da temperatura no comportamento de dispositivos fotônicos, foram analizadas duas estruturas. $\mathrm{Na}$ primeira, nós consideramos uma fibra óptica que exibe uma dispersão cromática muito baixa e extremamente plana para uma extensa faixa de comprimentos de onda [3]. Neste caso a formulação apresentada resumidamente na seção II, foi utilizada na obtenção de autovalores, reduzindo-se a solução de um problema do tipo: $[A]\{\phi\}=n_{\text {eff }}^{2}[B]\{\phi\}$. Aqui, $[A]$ e $[B]$ representam matrizes esparsas e complexas. Nesta simulação a janela computacional usada foi $9 \mu \mathrm{m}$ (direção $x$ ) e $9 \mu \mathrm{m}$ (direção $y$ ) dividida em aproximadamente 12.000 elementos quadráticos. Resultados preliminares mostraram que o uso de malhas com um número elementos maior, não melhora a precisão dos resultados numéricos. Em todas as simulações os parâmetros das fibras mostradas na Fig. 1a e 1b foram: $\eta_{1}$ $=1,05214, \eta_{2}=1,0, r_{1}=0,2 \mu \mathrm{m}$ e $r_{2}=1,8 \mu \mathrm{m}$. A dispersão cromática para várias temperaturas é mostrada na Fig. 2. Podese notar uma depedência linear da dispersão em função da temperatura, por exemplo, se nós considerarmos a dispersão cromática para $\lambda=1,53 \mu \mathrm{m}$ (Fig. imersa), a mesma varia linearmente com um coeficiente de temperatura de $6,66 \times 10^{-4}$ $\mathrm{ps} / \mathrm{nm} . \mathrm{km} / \mathrm{K}$.

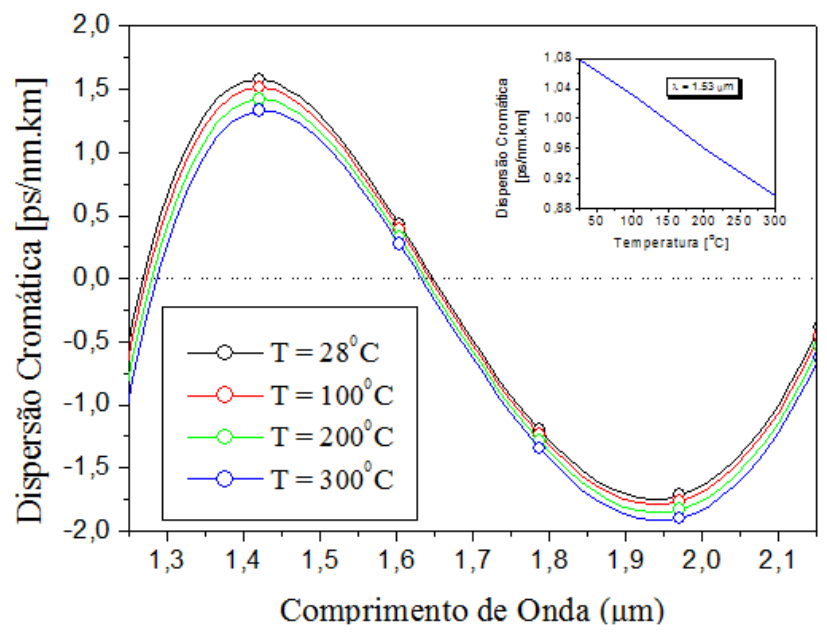

Fig. 2. Variação da Dispersão Cromática em função da Temperatura.

Este coeficiente é pequeno se comparado com outros perfis de índice de refração ou mesmo dos materiais [13], uma explicação seria pelo fato de uma fração da energia contida nos campos permanecerem na região central do núcleo (ar), o qual mantem seu índice de refração neste trabalho.

Na segunda aplicação, nós consideramos a estrutura mostrada na Fig. 1b. Aqui, foi analizado as características de acoplamento, em vários comprimentos de onda. A transferência de energia pode ser controlada através da variação da distância entre os núcleos, ou através da variação do comprimento de onda.

Neste trabalho, consideramos também a variação na temperatura e analisamos o efeito desta variação na tranferência de energia. Nas simulações nós consideramos a distância entre os núcleos, $d=0,5 \mu \mathrm{m}$. Os resultados foram obtidos para o comprimento de onda, $\lambda=1,53 \mu \mathrm{m}$. Nesta simulação, a janela computacional usada, também foi de $9 \mu \mathrm{m}$ (direção $x$ ) e $9 \mu \mathrm{m}$ (direção y) coberta por aproximadamente 12.000 elementos quadráticos para um passo de propagação $\Delta z=0,1 \mu \mathrm{m}$. Os resultados obtidos estão mostrados na Fig. 3, com a fibra do lado esquerdo, Fig. 1b, excitada com um feixe correspondente ao modo $E_{11}^{x}$, para o qual o índice de refração efetivo $\left(n_{\text {eff }}\right)$ é calculado através da relação $\beta / K_{0}$, obtido através da análise modal [8].

A distância de acoplamento obtida através do Método da Propagação Vetorial de feixes (VBPM) está de acordo com a distância obtida usando-se a análise modal através da relação $L_{B}=\pi /\left(\beta_{\text {eff } 1}-\beta_{\text {eff } 2}\right)$ [8], onde $L_{B}$ é o comprimento de batimento, $\beta_{\text {eff } 1}$ e $\beta_{\text {eff } 2}$ correspondem à constante de propagação dos modos super-simétrico e anti-simétrico de ordem mais baixa. A Fig. 3 mostra a região ampliada de máxima transferência de energia entre os núcleos das fibras do acoplador da Fig. 1b. A máxima transfência de energia para a temperatura de $28^{\circ} \mathrm{C}$ ocorre para a distância de acoplamento ao redor $444,5 \mu \mathrm{m}$, enquanto que para a temperatura de $300^{\circ} \mathrm{C}$ a máxima transferência de energia ocorre ao redor de $446 \mu \mathrm{m}$. Ou seja, ocorre uma variação de aproximadamente $1,5 \mu \mathrm{m}$ entre a distância de acoplamento considerando as temperatura de $28^{\circ} \mathrm{C}$ e $300^{\circ} \mathrm{C}$.

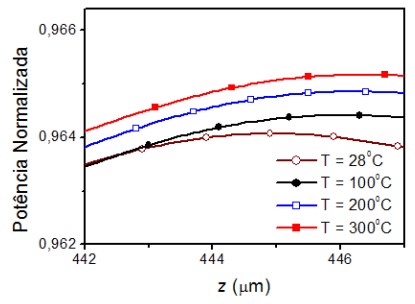

(a)

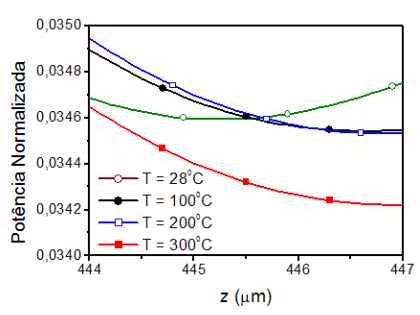

(b)
Fig. 3. Distância de Máxima tranferência de energia para: (a) núcleo 2 da Fig 1 b e (b) núcleo 1 da Fig 1 b.

A explicação para as pequenas variações nas características de acoplamento pode estar no fato de que a máxima transferência de energia ocorre em uma distância de acoplamento muito pequena, visto que os efeitos da temperatura, nas características de dispersão, podem envolver longas distâncias. A fibra proposta suporta dois modos propagantes cujas constantes de propagação tem diferenças significativas dificultando 
uma possível transferência de potência entre esses modos propagantes. Aplicações envolvendo sensores térmicos estão sendo analisadas, mas a presença do núcleo de ar reduz a sensibilidade da fibra com as variações de temperatura. Entretanto, testes preliminares estão sendo realizados para avaliar aplicação desse modelo de fibra óptica em sensores ópticos, visto que a análise mostrada neste trabalho foi feita focando apenas a influência da temperatura na transferência de energia.

A Fig. 4 mostra a variação do campo magnético, $h_{x}$ na direção de propagação, observa-se que o feixe de luz lançado na fibra esquerda da Fig. 1b, em $z=0 \mu \mathrm{m}$, é transferida para a fibra da direita. Com a continuação da propagação, essa transferência de energia acontece periodicamente ao longo do comprimento do acoplador.

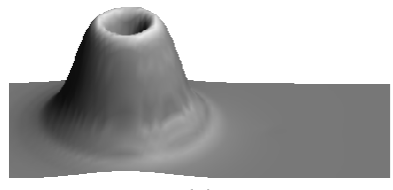

(a)

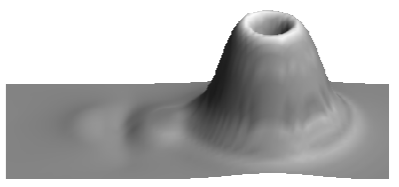

(b)
Fig. 4. Variação da componente principal do modo fundamental, $\mathrm{T}=28^{0} \mathrm{C}$ com polariação $X$ para: (a) $z=0 \mu \mathrm{m}$ e (b) $z=444,5 \mu \mathrm{m}$.

É importante ressaltar, que para obter uma faixa maior de comprimentos de ondas com dispersão cromática ultra-plana, nós estamos trabalhando na otimização da estrutura mostrada na Fig. 1A, usando técnicas evolutivas, tais como Algoritmos Genéticos em conjunto com método da Propagação Vetorial de Feixes [9].

\section{Conclusões}

Neste trabalho, o método dos elementos finitos em conjunto com a análise modal e o método da propagação vetorial de feixes, foi aplicado com sucesso para análise de estruras fotônicas, considerando variações na temperatura. A estrutura analizada exibe uma dispersão cromática ultra-plana, e esta foi obtida numericamente usando-se uma eficiente formulação baseada no método dos elementos finitos que inclui a dispersão no material. Os resultados obtidos mostram uma variação linear da dispersão cromática em função da variação da temperatura para um comprimento de onda $1,53 \mu \mathrm{m}$. Verificouse também, que as características de acoplamento sofrem variações pequenas com o aumento ou a diminuição da temperatura. Outros dispositivos encontram-se em análise e novos resultados serão apresentados durante a conferência.

\section{AGRADECIMENTOS}

Os autores agradecem a FAPERN e ao CNPq (563271/20086) pelo suporte financeiro, bem como a UFERSA pelo apoio estrutural.

\section{REFERÊNCIAS}

[1] A. Eason, B. Noble, e I. N. Sneddon, "On certain integrals of LipschitzHankel type involving products of Bessel functions", Phil. Trans. Roy. Soc. London, vol. A247, pp. 529-551, Abril 1955.
[2] V. Padgaonkar, A. Arbor, M. Lipson, e S. Pradhan, "Thermal effects in silicon based resonant cavit devices", http://www.nnin.org/doc/2004NNINreuVaidehee.pdf.

[3] J. P. da Silva, V. F. Rodríguez-Esquerre, H. E. Hernández-Figueroa, "Step index holey fiber for ultra-flattened chromatic dispersion", Proceedings of Integrated Photonics Research and Applications IPRA2006, Connecticut, 2006.

[4] J. Clerk Maxwell, "A Treatise on Electricity and Magnetism", 3rd ed. vol. 2. Oxford: Clarendon, pp. 68-73, 1892.

[5] B. J. Frey, D. B. Leviton, e T.J. Madison, "Temperaturedependent refractive index od silicon and germanium", http://arxiv.org./abs/physics/0606168.

[6] D. Correia, V. F. Rodríguez-Esquerre, e H. E. Hernández-Figueroa, "Genetic-algorithm and finit-element approach to the synthesis of dispersion-flattened fiber", Microwave and Opt. Technol. Lett, 32, 245248, 2001.

[7] K. Saitoh e M. Koshiba, "Chromatic dispersion control in photonic crystal fiber: application to ultra-flattened dispersion", Opt. Express 11, 843-852, 2003.

[8] H. E. Hernández-Figueroa, F. A. Fernández, Y. Lu e J. B. Davies, "Vectorial finite element modelling of 2D leaky waveguides" , IEEE Trans. Magn. 31, 1710-1712, 1995.

[9] D. Correia, J. P. da Silva e H. E. Hernández-Figueroa, " Genetic algorithm and finit-element design of short single-section passive polarization converter", IEEE Photonics Technol. Lett, 15, 915-917, 2003.

[10] J. P. da Silva, H. E. Hernández-Figueroa e A. M. F. Frasson, "Vectorial finite-element BPM analysis for transverse anisotropic media", J. Lightwave Technol., 21, 567-576, 2003.

[11] J. Crank, J. and P. Nicolson, "A practical method for numerical evaluation of solutions of partial differential equations of the heat conduction type", Proc. Camb. Phil. Soc. 43: 50-67, 1947.

[12] J. W. Fleming, "Material dispersion in lightguide glasses", Electron. Lett., 14 pp. 326-328, 1978.

[13] G. Ghosh, M. Endo, and T. Iwasalu, "Temperature-dependent sellmeier coefficients and chromatic dispersions for some optical fiber glasses", J. Lightwave Technol, 12, 1338-1342, 1994. 